\title{
Proposta de uma Aplicação web para Gestão e Simplificação do Processo de Doação para instituições de Caridade
}

\author{
Gabriel Silva \\ Ciência e Tecnologia \\ Instituto Federal de Educação Baiano \\ Senhor do Bonfim, BA, Brasil \\ gabrieljsilva2301@gmail.com \\ Marcos Souza \\ Ciência e Tecnologia \\ Instituto Federal de Educação Baiano \\ Senhor do Bonfim, BA, Brasil \\ marcos187if@gmail.com
}

\author{
Lethícia Souza \\ Ciência e Tecnologia \\ Instituto Federal de Educação Baiano \\ Senhor do Bonfim, BA, Brasil \\ lethicians12@gmail.com \\ Cleisson Batista \\ Ciência e Tecnologia \\ Instituto Federal de Educação Baiano \\ Senhor do Bonfim, BA, Brasil \\ cleissonf@gmail.com
}

\begin{abstract}
The present article refers to the construction of a web application named Doai, capable of managing and promoting donations for non-governmental organizations (NGOs) and charities in the Piemonte Norte do Itapicuru - Bahia. From interviews with institutions' managers, it became evident the lack of basic resources to maintain the continuity of the performed activities, It was also found that in many cases donations do not reflect your real needs. This demonstrates the lack of information between donors and these institutions. The problems experienced in these organizations was motivate the development this proposal, which aims to promote existing institutions in the region, with the modernization of dissemination actions, with the simplification of the donation process and with the resources available to leverage the contributions. After construction, the tool was submitted to analysis, with the participation of 73 (seventy-three) people from the region. In this research it was possible to realize that few people are involved with philanthropic activities. On the other hand, a large part of the public are motivated to donate using the tool, showing out how it offers good usability. In addition, much of the public also shows interest in virtual payment methods. From a technical point of view, the tool has a good user acceptance and meets the proposed requirements.
\end{abstract}

\section{KEYWORDS}

Instituições de Caridade, Doações, Sistema Web

\section{CONTEXTUALIZAÇÃO}

As entidades filantrópicas são sociedades sem fins lucrativos que tem o objetivo de produzir o bem, seja assistindo à família, à maternidade, à infância ou até mesmo ajudando animais vítimas de maus tratos [1]. O cenário atual de desemprego, em que 12,5 milhões de pessoas estão desempregadas [2], aliado às necessidades de atendimento à população, torna ainda mais interessante o papel destas entidades, que agem como um mecanismo de transferência de renda e atendimento às demandas populares.

As doações representam a base de sustentação destas organizações. Entretanto, constatou-se através de pesquisa realizada na cidade de Senhor do Bonfim, que os gestores destas instituições enfrentam dificuldades para manutenção desses serviços, tendo em vista, que na maior parte dos casos as doações não estão em consonância com as necessidades das mesmas. Em um caso hipotético, para exemplificar esse tipo de situação vivenciada, supondo que a demanda seja por insumos para limpeza e alimentação, neste momento, surgem doações de roupas. Vale ressaltar, que apesar de importante esse tipo de item, necessidades básicas deixam de ser supridas. Em todos os casos pesquisados, os gestores relataram que os colaboradores (voluntários ou funcionários) destas instituições se dispõem a cobrir despesas para manutenção destas demandas básicas.

Essa situação expõe a necessidade de melhoria nos instrumentos de captação de recursos e de conscientização das pessoas. Assim, dada as necessidades urgentes destas instituições, surgiu a ideia de criação de uma ferramenta web para controle e gerenciamento de doações. Esta ferramenta, intitulada doaí, tem como papel aproximar as instituições de caridade presentes no Território de Identidade Piemonte Norte do Itapicuru da sociedade, facilitando o processo de doação e permitindo a emissão de comprovantes para abatimento na declaração do Imposto de Renda.

Nesta ferramenta os administradores podem cadastrar campanhas de publicidade, de acordo com as necessidades das instituições, para que fiquem visíveis na plataforma (possibilitando o compartilhamento nas mídias sociais) e para que sejam também encaminhadas através de notificações por e-mail para os doadores que autorizaram o envio destas informações.

\section{METODOLOGIA}


O desenvolvimento do sistema utilizou o ciclo de vida em cascata. Apesar deste ciclo de vida ser menos flexível, já que as etapas do processo seguem um fluxo sequencial e há uma dependência da finalização da fase anterior para prosseguimento da seguinte[3], esta escolha surgiu naturalmente na equipe, devido tanto a simplicidade do projeto quanto ao próprio processo de execução da disciplina ao qual a proposta estava inserida.

A construção do projeto seguiu de forma minuciosa as etapas do ciclo de vida do processo de desenvolvimento de sistemas. Em linhas gerais, as etapas foram as seguintes: elicitação e análise de requisitos, projeto de software, implementação, validação e implantação[3]. A elicitação de requisitos teve como objetivo compreender as necessidades dos gestores dessas organizações e as principais regras de negócio do sistema a ser desenvolvido. Para isso foram utilizadas entrevistas semiestruturadas. Durante essa etapa foram construídos documentos de descrição de requisitos e o modelo de casos de uso que apresenta os requisitos funcionais, suas relações e os atores responsáveis por essas interações[4].

A figura 1 apresenta o diagrama de caso de uso da ferramenta doai. Este modelo apresenta as principais funcionalidade do sistema na perspectiva do cliente [3]. Os atores identificados neste modelo foram: o administrador, o gerente da ONG e o doador. O administrador possui a atribuição de autorizar o cadastro de gestores de ONGs, gerenciar novas instituições de caridade e realizar o cadastramento de notícias e campanhas. Todas essas responsabilidades são denominadas de casos de uso do administrador. O doador teria a função de realizar o seu cadastro no ambiente, realizar doações e gerar declarações das suas contribuições. O gestor da ONG por sua vez, teria como atribuição a realização do cadastro na plataforma e a solicitação da inclusão da instituição no ambiente.

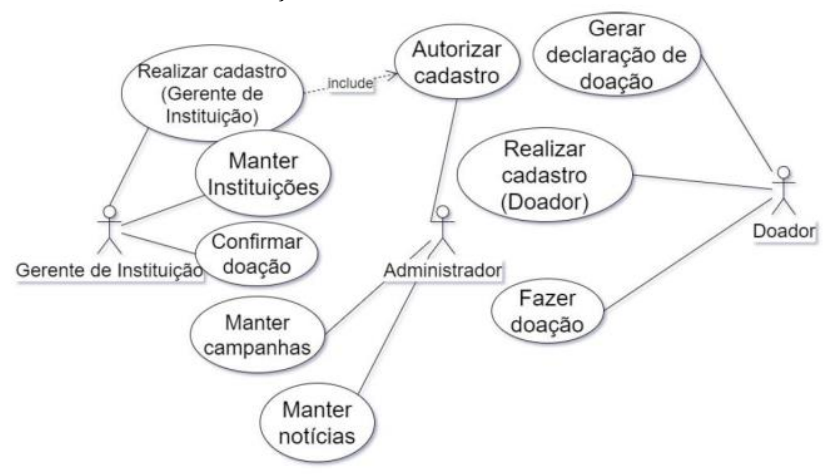

Figure 1. Diagrama de caso de uso

Para validação dos requisitos, foram criados protótipos de interface. Essa abordagem também possibilitou uma melhor compreensão do software a ser desenvolvido e facilitou as discussões sobre as estratégias de desenvolvimento. Esse primeiro passo subsidiou informações para o processo de modelagem, onde foram elaborados diagramas de atividade, de classe, de entidade e relacionamento (ER) e lógico. Todos esses modelos foram construídos na ferramenta de modelagem Draw. Alguns destes modelos serão descritos nos próximos parágrafos.

O diagrama ER, que descreve os dados do negócio na visão do usuário[4] é apresentado na Figura 2. Nele são destacados os objetos (entidades), seus atributos e seus relacionamentos [4].

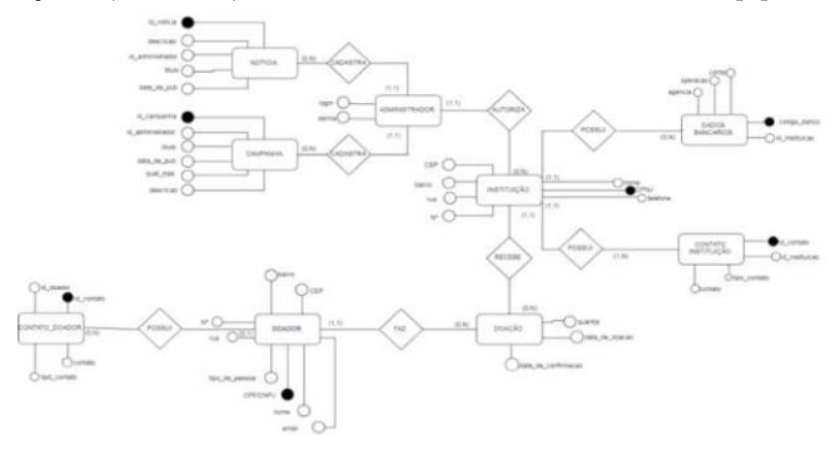

Figure 2. Modelo ER

Uma versão mais detalhada deste diagrama pode ser encontrado em

https://drive.google.com/open?id=lddxrplMFdgq0HDR76MdhW2 yvmPr9jqw. A partir da criação deste diagrama foi elaborado o modelo lógico de banco de dados, apresentado na Figura 3. Este por sua vez é uma representação específica de um banco de dados, que leva em consideração as regras de negócio, padrões e nomenclaturas adoptadas pelos SGBDs [4].

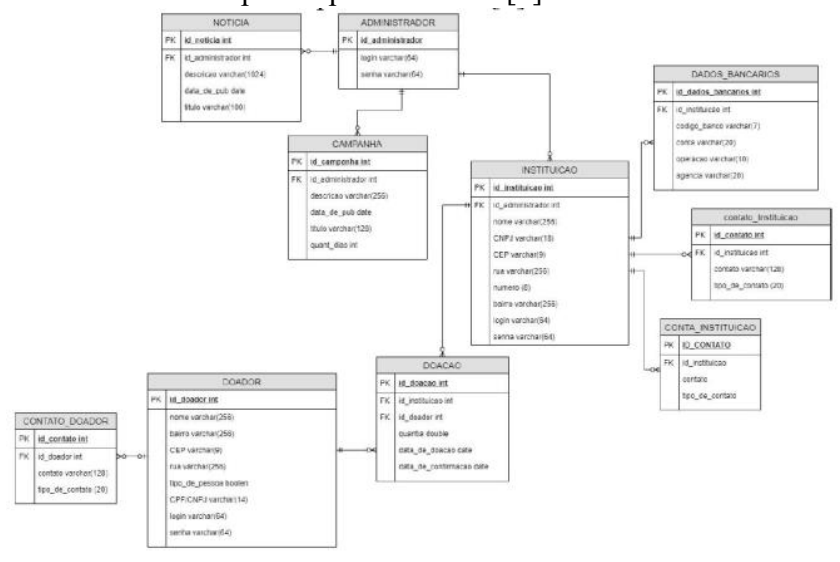

Figure 3. Modelo Lógico

Uma versão mais detalhada deste diagrama pode ser encontrado em https://drive.google.com/open?id=lWVVNZ9YXNbcufOGIAEZQIWWUUiM7A. Ao final da modelagem deu-se início ao processo de desenvolvimento da aplicação, que foi dividida em duas etapas. $\mathrm{Na}$ primeira etapa ocorreu a implementação da API (Application Programming Interface) e na etapa seguinte iniciou-se o desenvolvimento da interface do usuário. A API ficou responsável pelo gerenciamento das regras de negócio da aplicação, fazendo a comunicação com o banco de dados e o processamento das informações. Essa camada 
foi escrita na linguagem JavaScript utilizando o interpretador NodeJs. O sistema gerenciador de banco de dados (SGBD) utilizado foi o mongoDB, que apesar de ser um banco de dados não relacional, a curva de aprendizagem deste SGBD costuma ser menor em relação aos bancos relacionais, que utilizam a linguagem SQL. Além disso, este SGBD proporciona uma maior escalabilidade, já que diminui os impactos no sistema ao fazer alterações nas regras de negócio [5].

Ainda assim, o diagrama ER criado não representou perda de tempo ou esforço inútil, pois através dele foi possível gerar melhor compreensão entre os envolvidos sobre o que e como desenvolver. Esta "documentação" representou valor ao processo de desenvolvimento. É interessante frisar que a decisão de implementar o software em camadas bem definidas e separadas garantiu tal coesão ao código e que a mudança na escolha do SGBD afetou apenas a camada de persistência. Tem-se aí um exemplo de como as boas práticas no projeto de software permitem um menor acoplamento.

Para a interface, foram utilizados os Framework VueJS e o Vuetify. O VueJS é um framework progressivo da linguagem JavaScript, utilizado na criação de interface (de usuário) centrado na criação de componentes reutilizáveis [6]. Enquanto o Vuetify é uma biblioteca que fornece uma gama de componentes para o VueJs, baseado no material design do google, segundo sua documentação oficial.

Finalizado o desenvolvimento, foram realizadas baterias de testes a fim de garantir que o produto desenvolvido atendesse aos requisitos elicitados. O processo de testes se baseou na simulação do uso da aplicação por um usuário normal pelos participantes do projeto, na qual cada um dos participantes testou cada requisito do sistema. Ao final das sessões de teste, os poucos erros de lógica e interface encontrados foram rapidamente corrigidos e então testados novamente até que o software finalmente pudesse ser finalizado.

\section{RESULTADOS OBTIDOS}

A partir da implementação dos requisitos e também da integração da interface com a API, foi possível conceber a aplicação Doai. A aplicação, além de facilitar o processo de doação e gerenciamento das mesmas, foi capaz de gerar comprovantes de doações, para utilização em declarações de imposto de renda. A aplicação é responsiva, ou seja, se adapta a tela de qualquer dispositivo, seja smartphone, tablet ou desktop. A figura 4 demonstra a tela inicial da aplicação.

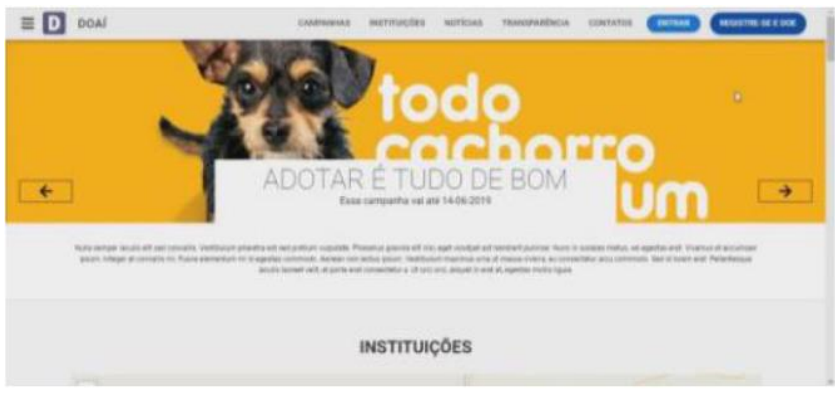

Figure 4. Interface da página inicial da aplicação Doai

Com a simulação de um ambiente de produção, foi realizada uma pesquisa com 73 (setenta e três) pessoas, a fim de verificar a viabilidade de implantação da aplicação e também obter novos requisitos na perspectiva do doador.

A partir desta pesquisa foi constada que a maioria das pessoas não se sentem muito engajadas em relação a atividades solidárias. Como demonstrado no gráfico, 1,36 porcento dos entrevistados se sentem pouco engajados e 46,7 porcento se sentem razoavelmente engajadas com estas atividades.
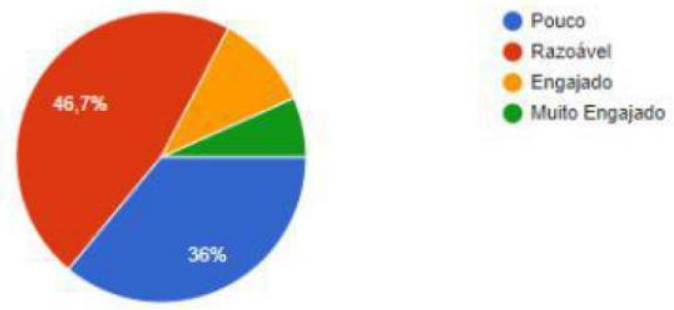

Graphic 1. Engajamento dos usuários com atividades solidárias

Um indício desse pouco engajamento pode estar relacionado ao fato de a maioria dos entrevistados não conhecerem instituições de caridade na região, como demonstra o gráfico 2, na qual 60 porcento dos entrevistados afirmaram não conhecer nenhuma instituição de caridade na região.

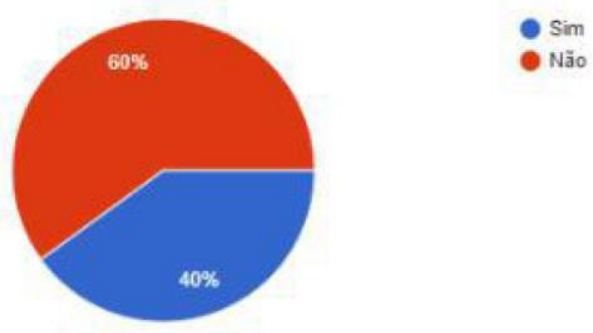

Graphic 2. Usuários que conhecem instituições de caridade na região

A pesquisa demonstrou também que a maioria dos entrevistados, cerca de 94,7 porcento, afirmaram se sentirem motivados a 
doarem utilizando a aplicação doai, como demonstra o gráfico 3. $\mathrm{O}$ que pode ser um indicativo de que a plataforma pode potencializar o número de doações.

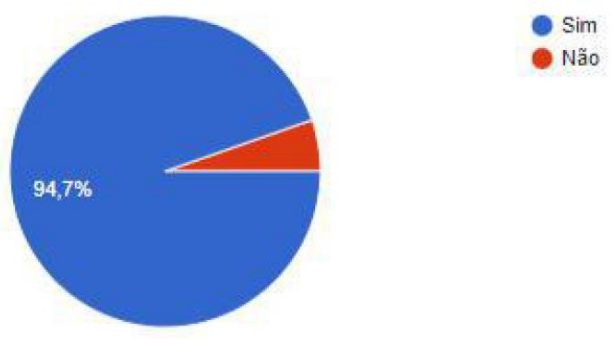

\section{Graphic 3. Usuários estimulados a doar utilizando a ferramenta}

Além disso, como demonstrado no gráfico 4, cerca de 88 porcento dos entrevistados acharam o site fácil de ser utilizado, evidenciado a boa usabilidade da aplicação.

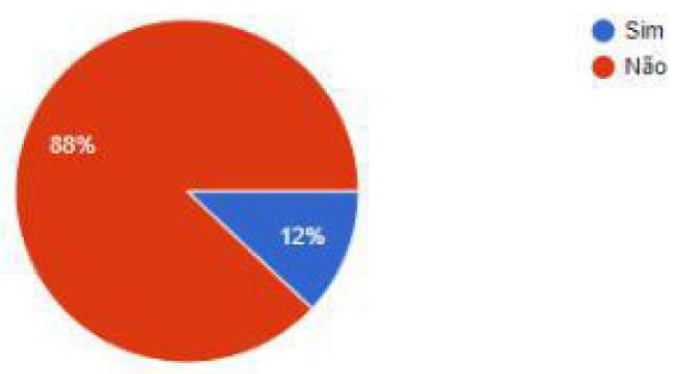

Graphic 4. Gráfico da pesquisa realizada com potenciais doadores

Além do meio tradicional de doação adotada pela aplicação (transferência bancária) os entrevistados também demonstraram interesse em métodos alternativos para realizar as doações, as mais citadas foram respectivamente: cartão de crédito, aplicativos de pagamento online e boleto. Um dos entrevistados sugeriu a adição de um novo recurso para facilitar ainda mais o processo de transferência, "Poderia deixar um QR code pra quem desejasse pagar com Nubank, ou até mesmo via PicPay". Esta pesquisa serviu para avaliar a ferramenta, verificar possíveis falhas e identificar recursos que poderão ser implementados em versões futuras.

\section{CONCLUSÃO}

O desenvolvimento da aplicação foi um grande desafio, desde o processo de levantamento de requisitos até a escolha das tecnologias e a programação. Contudo, tendo em vista as necessidades urgentes destas instituições e o impacto que a ferramenta pode gerar, percebe-se a relevância da aplicação Doai, que possui potencial para alavancar as doações. Dentre os recursos principais é possível destacar: envio de notificações por e-mail, publicização das campanhas e notícias e possibilidade de compartilhamento de campanhas em mídias sociais. Outras funcionalidades, que tem por objetivo gerenciar as doações efetuadas, também são úteis, tanto para os doadores quanto para as instituições, já que a aplicação dispõe de um histórico no painel de controle do usuário. Além disso, a aplicação possui uma seção exclusiva para auditoria de doações, que tem por objetivo, não só evitar fraudes, como também tornar a aplicação mais transparente, ganhando assim, maior confiabilidade. $\mathrm{O}$ usuário também tem a possibilidade de visualizar as instituições mais próximas de sua localização através de recursos de mapa. A junção destas funcionalidades simplifica o processo de doação, e contribuem para uma maior visibilidades das instituições da região. Apesar de o software ser gratuito, o código se encontra fechado para melhorias e adição de novos requisitos que podem surgir posteriormente.

\section{APÊNDICE}

\section{Perguntas da Última Pesquisa:}

1. Você conhece alguma instituição de caridade na cidade de Senhor do Bonfim ou região?

2. Você conhece alguma ferramenta (site ou aplicativo) que facilite o processo de doação para ONGs e Instituições de caridade?

3. Se sim, já utilizou alguma dessas ferramentas?

4. Quão engajado você se sente em relação à atividades solidárias?

5. Você se sente motivado a doar utilizando um site como o Doaí?

6. Você compartilharia campanhas de doação ou instituições cadastradas no site em suas redes sociais?

7. A partir da visualização do vídeo, você achou o site díficil de usar?

8. Se você fosse utilizador do site, gostaria de receber novas campanhas de doação por email?

9. Qual sua opinião sobre o recurso de notificação?

10. Você acha que deveria existir outros métodos de doação? Se sim, quais?

\section{REFERÊNCIAS}

[1] Revista Filantropia. 2008. Qual o conceito de entidade filantrópica? Recuperado em https://www.filantropia.ong/ informacao/2427qual_o_conceito_de_entidade_fila ntropica

[2] UOL. 2019. Desemprego é de $11,8 \%$ e atinge 12,5 milhões; informalidade tem novo recorde. Recuperado de https://economia.uol.com.br/empregosecarreiras/ noticias/redacao/2019/10/31/de semprego-pnad-ibge.htm

[3] Ian Sommerville. 2011. Engenharia de Software. (9th. ed.). Pearson Addison Wesley, São Paulo.

[4] Elaini Angelotti. 2010. Banco de Dados. (1st. ed.). Editora do Livro Técnico, Curitiba.

[5] Daniela Beilich. 2016. MONGODB: ALTO DESEMPENHO E ESCALABILIDADE EM BANCO DE DADOS. Recuperado de https://www.zalts.com.br/mongodb-alto-desempenho-e-escalabilidadeembanco-dedados/

[6] Adam Freeman. 2018. Pro VueJS. (1st. ed.). Apress Berkeley, CA. 